\title{
Does frozen section increase false negativity in cervical mediastinoscopy during mediastinal lymph node staging compared to paraffin section?
}

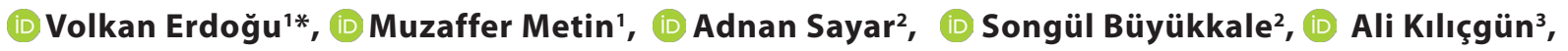 \\ Serda Kanbur Metin ${ }^{4}$, $\square$ Atilla Pekçolaklar ${ }^{5}, \square$ Necati Çıtak $^{6}, \square$ Halide Nur Ürer ${ }^{7}, \square$ Atilla Gürses ${ }^{1}$
}

'Department of Thoracic Surgery, University of Health Sciences, Yedikule Chest Diseases and Thoracic Surgery Education and Research Center, Istanbul, Turkey

${ }^{2}$ Department of Thoracic Surgery, Memorial Şişli Hospital, Istanbul, Turkey

${ }^{3}$ Department of Thoracic Surgery, Bolu Abant İzzet Baysal University, Faculty of Medicine, Bolu, Turkey

${ }^{4}$ Department of Thoracic Surgery, University of Health Sciences, Süreyyapaşa Chest Diseases and Thoracic Surgery Training and Research Hospital, Istanbul, Turkey

${ }^{5}$ Department of Thoracic Surgery, Bursa City Hospital, Bursa, Turkey

${ }^{6}$ Department of Thoracic Surgery, University of Health Sciences, Bakırköy Dr. Sadi Konuk Training and Research Hospital, Istanbul, Turkey ${ }^{7}$ Department of Pathology, University of Health Sciences, Yedikule Chest Diseases and Thoracic Surgery Education and Research Center, Istanbul, Turkey

\begin{abstract}
Background: Cervical Mediastinoscopy (CM) is the most important invasive method in mediastinal lymph node staging. Lymph nodes dissected via CM can either be sent to frozen section (FS) for analysis allowing thoracotomy to be performed on the same session, or to paraffin section (PS) where thoracotomy is performed as a separate session (as a separate operation). This study compares the falsenegative ratio of mediastinoscopic FS and PS analysis.
\end{abstract}

Materials and Methods: 454 patients with primary lung cancer who have undergone CM between January 2003 and December 2005 have been retrospectively analysed. This study evaluates whether FS analysis increases the false-negative rate of lymph node biopsies.

Results: 160 cases from the PS group and 113 cases from the FS group were included in the study. The mean age of the patients was 56.4 years (range 28-77 years). There were 260 men and 13 women. In the PS group, mean thoracotomy time after CM was 9.9 days. False negativity of CM in the PS and the FS groups was found to be $9.2 \%$ and $8 \%$, respectively.

Conclusion: There was no statistically significant difference in terms of false negativity between FS and PS in mediastinal staging $(\mathrm{p}=0.598)$.

Keywords: Frozen section, paraffin section, lung cancer staging

Corresponding Author*: Volkan Erdoğu, MD. Yedikule Chest Diseases and Thoracic Surgery Training and Research Hospital, Kazlicesme Mh, Belgrade Kapi yolu Cad No: 1, 34020, Zeytinburnu / Istanbul / Turkey.

E-mail: verdogu@gmail.com Phone: +90 2124090200

Doi: $10.26663 /$ cts.2020.0005

Received 04.09.2019 accepted 06.11.2019 


\section{Introduction}

The most important criteria in determining treatment modality and disease prognosis in lung cancer without distant metastasis is, the state of the mediastinal lymph nodes [1-2]. Cervical mediastinoscopy (CM) accepted as the gold standard method for invasive mediastinal staging. $\mathrm{CM}$ has $78 \%$ specificity, $100 \%$ sensitivity and $91 \%$ negative predictive value (NPV) in mediastinal staging [3]. The biggest disadvantage of CM is, the procedure's false negativity rate. In previous studies this rate has been found to be $1-10 \%$ [4]. Non-surgical treatment is the primary modality for patients determined to have positive mediastinal lymph nodes (pN2) using CM $[5,6]$. Appropriate mediastinal staging reduces the number of patients undergoing unnecessary thoracotomy. Indications for CM are defined as; lymph node greater than 1 $\mathrm{cm}$ on computed tomography $(\mathrm{CT})$, positive mediastinal and/or hilar lymph node on Positron Emission Tomography-Computed Tomography (PET-CT), central or large peripheral tumor and a histology of adenocarcinoma [7].

The aim of this study is to evaluate whether there is a significant difference between the false- negativity rates of frozen section (FS) and paraffin section (PS) methods in $\mathrm{CM}$ lymph node staging in patients diagnosed with primary lung cancer without distant metastasis.

\section{Materials and Methods}

454 patients diagnosed with primary lung cancer undergone CM or thoracotomy on the same or different sessions were retrospectively recorded between January 2003 - December 2005 in Yedikule Chest Diseases and Thoracic Surgery Education and Research Hospital. All of the patients were diagnosed and clinically staged with radiological and broncological procedures. Patients without mediastinal lymph node metastasis (pN0), were surgically treated with a lobectomy on the same session or at least on one of the next sessions. The sampled lymph nodes were staged with The American Joint Committee on Cancer (AJCC) staging system revised in 2009, as recommended by Mauntain [8]. The decision whether thoracotomy should be made on the same session or a different session was made by the surgeons. All of the patients who were treated in our clinic gave informed consent by signing a statement allowing the use of their data for clinical trials. The study was approved by the local institutional review board (2020-
240-01) and was conducted in accordance with the principles of the Declaration of Helsinki.

In FS group, lymph nodes were sent to the pathology laboratory without fixation. Lymph nodes were analysed by both imprinting and by freezing methods making 1 or 2 slices. Frozen section results were reported after an average of 25 minutes. In PS group, the lymph nodes sent by fixed in formaldehyde then added to paraffin blocks by pathologists and were analysed after being dissected into at least 3 slices. Both FS group and PS group, the specimens were evaluated by 3 pathologists who were specialized in lung cancer in our clinic. Immunohistochemical staining was not used in any of the patients. The patients that were found to be pNO on the FS analysis were treated with thoracotomy on the same session, while patients who were found to be pN0 with PS were treated with thoracotomy after a mean of 9.9 days. The patients diagnosed as pN2 and/ or $\mathrm{pN} 3$ in the opposite mediastinum were referred to oncology departments for adjuvant or neoadjuvant therapy. European Society of Thoracic Surgeons (ESTS) guideline published in 2004 was taken as reference in intraoperative mediastinal staging methods due to the differing preferences of the surgeons whether to perform systemic mediastinal lymph node dissection or a biopsy of lymph nodes solely in thoracotomy. Hence, it was obligatory to sample at least 3 mediastinal lymph nodes with one being the subcarinal (number 7) station in the lobe's drainage pathway [9]. The standard cervical mediastinoscopy method was used in the study. Video-assisted mediastinoscopic lymphadenectomy (VAMLA) or videomediastinscopy methods were not used during the study time in those years.

55 patients (12 PS, 43 FS) were excluded from the study because they couldn't be intraoperatively staged so their false negativity in CM couldn't be analysed. 59 patients were excluded from the study for receiving neoadjuvant therapy. 24 patients although reported as pNO in CM, were either operated on in another clinic or refused surgical treatment, 33 cases who could only be surgically explored during thoracotomy due to tumor size and local invasion, were excluded from the study. 9 cases were excluded from the study due to complications (haemorrhage, severe arrhythmia, etc.) that occurred during $\mathrm{CM}$ causing insufficient mediastinal lymph node sampling and 1 case was excluded from 
the study because of peroperative exitus. According to these criteria, a total of 181 patients were excluded from the study, and a total of 273 patients, of whom 160 were from the PS group and 113 were from the FS group were included in the study.

\section{Statistical Analysis}

The data were entered in the Statistical Package for the Social Sciences (SPSS 23.0 version for Windows; SPSS Inc., Chicago, Illinois, United States). Age; gender; pN status; has been analyzed. Student-t test was used in comparison of false negativity, sensitivity, specificity and negative predictive value results values between groups. Pearson's chi-square test was used for the analysis of qualitative variations. $\mathrm{p}<0.05$ value was considered statistically significant.

\section{Results}

The mean age of the patients included in the study was
56.4 years (range $28-77$ years). There were 260 men and 13 women. In the PS group, mean thoracotomy time after $\mathrm{CM}$ was 9.9 days. The $\mathrm{CM}$ sampling was reported as pN0 in 120 of 160 patients (75\%) and 40 patients (25\%) were reported as $\mathrm{pN} 2-3$. These patients were referred to the oncology department for adjuvant or neoadjuvant treatment. Among the patients who have undergone thoracotomy, 27 patients $(22.5 \%)$ who could not be diagnosed with invasive or non-invasive techniques prior to thoracotomy, were diagnosed as $\mathrm{pN} 2$ even though they were not expected to have single or multiple station positivity. Out of these $27 \mathrm{pN} 2$ patients, 11 (6.8\%) were missed due to false negativity of $\mathrm{CM}$, even though their lymph node stations could be easily accessed (2R-2L4R-7). The sensitivity, specificity, NPV and false negativity ratio of CM procedure with PS was recorded as $82.2 \%, 100 \%, 90.8 \%$, and $9.2 \%$ respectively (Table 1 ).

Table 1. FS and PS group false negativity, sensitivity and specificity, negative predictive value results.

\begin{tabular}{|c|c|c|c|c|c|c|c|}
\hline Method & $\begin{array}{l}\text { Number of } \\
\text { patients }\end{array}$ & $\mathrm{CM}$ pNO & $\mathrm{CM}$ pN2-N3 & $\begin{array}{l}\text { Thoracotomy pN2 (4R-7) } \\
\text { False \% }\end{array}$ & Sensitivity $\%$ & Specificity \% & NPV \% \\
\hline FS & 113 & 75 & 38 & 8 & 88 & 100 & 92 \\
\hline PS & 160 & 120 & 40 & 9.2 & 82.2 & 100 & 90.8 \\
\hline$P$ value & & & 0.120 & 0.598 & & & \\
\hline
\end{tabular}

75 of the 113 patients (64.4\%) in the FS group were found to be pN0. 38 (33.6\%) patients were reported as pN2-3 in CM and these patients were referred to Oncology Department for adjuvant or neoadjuvant treatment. Among all the patients who have undergone thoracotomy, 18 (24\%) who could not be diagnosed via invasive or non-invasive techniques prior to thoracotomy, were diagnosed as pN2. 6 of the 18 patients (5.3\%) were missed because of the false negativity of $\mathrm{CM}$, although the lymph node stations were accessible with CM (2R-2L-4R-4L-7). The sensitivity, specificity, NPV and false negativity ratio of CM procedure with FS was recorded as $88 \%, 100 \%, 92 \%$, and $8 \%$ respectively (Table 1).

Among patients who have undergone thoracotomy and diagnosed as $\mathrm{pN} 2$ in lymph node station number 7 , 4 patients (4/6-33\%) were from the FS group and 9 patients (9/11- 81.8\%) from the PS group. In both groups, 2 patients were found to be 4R false negative (2/6-33\% in the FS group, 2/11-18\% in the PS group) (Table 2).

Table 2. PS and FS false negativity in lymph node stations.

$\begin{array}{lllr}\text { Lymph node station } & \begin{array}{l}\mathrm{pN} 2 \text { diagnosed in thoracotomy following PS } \\ \text { (Number of Patients) }\end{array} & \begin{array}{l}\text { pN2 diagnosed in thoracotomy following FS } \\ \text { (Number of Patients) }\end{array} & >0.05 \\ 4 \mathrm{R} & 2 & 2 & >0.05 \\ 7 & 9 & 4 & >0.05\end{array}$

Abbrev.; 4R: right lower paratracheal lymph node, 7: subcarinal lymph node, FS: frozen section, PS: paraffin section 


\section{Discussion}

Mediastinal lymph node involving is the most important factor in defining the prognosis in lung cancer. $15-26 \%$ of the patients are found to be $\mathrm{pN} 2$ during thoracotomy in patients initially diagnosed as $\mathrm{pN} 0$ with invasive and non-invasive techniques. This group has the best survival rate among the pN2's. The treatment suggested to these patients is achieving the negative surgical margin (R0) followed by adjuvant therapy. 5 year survival rate is around 30\% [10]. In our study, although not expected in thoracotomy, we found $\mathrm{pN} 2$ in $22-24 \%$ of the cases, which is similar to the results in published literature.

In literature, false negativity in $\mathrm{CM}$ is between $1-10 \%$. The highest false negativity is found in subcarinal lymph nodes, and less frequently in the 4R station. Lamaire et al. have recorded a diagnosis of $\mathrm{pN} 2$ during mediastinoscopy as $23 \%$, and the false negativity ratio as $5.5 \%$ [11]. The false negativity ratio between PS and FS in our study was recorded less than $10 \%$ as in literature, and there was no statistically significant difference. Most frequent false negativity in both groups was seen in subcarinal lymph nodes.

The advantages of FS analysis include patients undergoing anesthesia only once, same session thoracotomy option and one time hospitalization which causes cost reduction. In a study where patients' opinions were taken, the result was that patients want to undergo CM thoracotomy on the same session. The disadvantage of FS analysis is, since it is a peroperative procedure, one slice is excised unless more is needed, and this could be a problem in identifying metastasis. Also, dysmorphology may occur due to the freezing of the piece. However, studies have shown that this is not a disadvantage. Moreover, the slice excised for analysis, even if only imprinting method is used, has found to be advantageous to that of FS because it saves time and there is more sample area to be analysed and also their false negativity ratio is similar. On the other hand in paraffin section analysis is made through three slices, unless more is needed, which is advantageous in identifying metastasis. However, studies have shown that there is no significant difference compared to FS analysis [12].

Our study had a few limitations of note. First, this was a single-center study based on a relatively small number of patients. Second, the patients underwent operations performed by several surgeons. Third, videomediastinoscopy wasn't used. Fourth, pathological examinations performed several pathologists. Fifth, our conclusions are based on a retrospective analysis of patient data. Accordingly, further researches into prospective designed studies are suggested.

In conclusion, mediastinal staging is one of the most important prognostic factors in non-small cell lung cancer patients. Mediastinal staging also provides a therapeutic strategy for patients. The cervical mediastinoscopy is still considered the gold standard in mediastinal staging. In this study, we investigated whether the FS method increases the false negativity of cervical mediastinoscopy compared to the PS method. We could not find any difference between the two groups. FS is a safe method for evaluating $\mathrm{CM}$ results.

\section{Declaration of conflicting interests}

The authors declared no conflicts of interest with respect to the authorship and/or publication of this article.

\section{Funding}

The authors received no financial support for the research and/or authorship of this article.

\section{References}

1. Vansteenkiste J, Crinò L, Dooms C, Douillard JY, Faivre-Finn C, Lim E et al. 2nd ESMO Consensus Conference on Lung Cancer: early-stage non-small-cell lung cancer consensus on diagnosis, treatment and follow-up. Ann Oncol 2014;25:1462-74.

2. Sezen CB, Gokce A, Kalafat CE, Aker C, Tastepe AI. Risk factors for postoperative complications and long-term survival in elderly lung cancer patients: a single institutional experience in Turkey. Gen Thorac Cardiovasc Surg 2019;67:442-9.

3. Silvestri GA, Gonzalez AV, Jantz MA, Margolis ML, Gould MK, Tanoue LT et al. Methods for staging non-small cell lung cancer: Diagnosis and management of lung cancer, 3rd ed: American College of Chest Physicians evidence-based clinical practice guidelines. Chest 2013;143: e211S-e250S

4. Detterbeck FC, DeCamp MM Jr, Kohman LJ, Silvestri GA. Lung cancer. Invasive staging: the guidelines. Chest 2003;123:167S-175S. 
5. Ferguson MK. Optimal management when unsuspected N2 nodal disease is identified during thoracotomy for lung cancer: cost-effectiveness analysis. J Thorac Cardiovasc Surg 2003;126:1935-42.

6. Sezen CB, Kocaturk C. I, Bilen S, C. Kalafat E, Aker C, Karapinar K. Long-term results of completion pneumonectomy for the non-small cell lung cancer. Acta Chir Belg 2019;119:303-8.

7. De Leyn P, Dooms C, Kuzdzal J, Lardinois D, Passlick B, Rami-Porta $\mathrm{R}$ et al. Preoperative mediastinal lymph node staging for non-small cell lung cancer: 2014 update of the 2007 ESTS guidelines. Transl Lung Cancer Res 2014;3:225-33.

8. Rusch VW, Asamura H, Watanabe H, Giroux DJ, Rami-Porta $\mathrm{R}$, Goldstraw P. The IASLC lung cancer staging project: a proposal for a new international lymph node map in the forthcoming seventh edition of the TNM classification for lung cancer. J Thorac Oncol 2009;4:568-77.
9. Lardinois D, De Leyn P, Van Schil P, Porta RR, Waller D, Passlick B et al. ESTS guidelines for intraoperative lymph node staging in non-small cell lung cancer. Eur J Cardiothorac Surg 2006;30:787-92.

10. Cerfolio RJ, Maniscalco L, Bryant AS. The treatment of patients with stage IIIA non-small cell lung cancer from N2 disease: who returns to the surgical arena and who survives. Ann Thorac Surg 2008;86:912-20.

11. Lemaire A, Nikolic I, Petersen T, Haney JC, Toloza EM, Harpole DH Jr et al. Nine-year single center experience with cervical mediastinoscopy: complications and false negative rate. Ann Thorac Surg 2006;82:1185-9.

12. Attaran S, Jakaj G, Acharya M, Anderson JR. Are frozen sections of mediastinoscopy samples as effective as formal paraffin assessment of mediastinoscopy samples for a decision on a combined mediastinoscopy plus lobectomy? Interact Cardiovasc Thorac Surg 2013;16:872-4. 